\title{
A CHARACTERIZATION OF HARMONIC FOLIATIONS BY VARIATIONS OF THE METRIC
}

\author{
MICHAEL D. HVIDSTEN AND PHILIPPE TONDEUR ${ }^{1}$
}

\begin{abstract}
In this paper it is shown that a harmonic Riemannian foliation of a Riemannian manifold can be characterized as being a critical point of the energy of the foliation under certain variations of the manifold's Riemannian metric. These variations are those induced by the flows of vector fields on the manifold.
\end{abstract}

1. Let $\mathscr{F}$ be a foliation on a Riemannian manifold $\left(M, g_{M}\right) . \mathscr{F}$ is said to be harmonic if all the leaves of $\mathscr{F}$ are minimal submanifolds [KT]. The existence of such a metric imposes strong conditions on $\mathscr{F}$ (Sullivan's tautness condition [S]). A foliation is Riemannian if the metric $g_{M}$ is bundle-like, i.e. the induced metric $g_{Q}$ in the normal bundle $Q$ is holonomy-invariant [R]. Such foliations were characterized in [KT] as critical points of the energy functional under a class of so-called special variations $\mathscr{F}_{t}$ of the foliation $\mathscr{F}_{0}=\mathscr{F}$, variations induced by exponentiating sections $s \in \Gamma Q$ in the metric corresponding to the modelling Riemannian manifold. In this note we show that harmonic foliations can be alternatively characterized as critical points of the energy functional under a certain class of variations $g_{M}(t)$ of the Riemannian metric $g_{M}(0)=g_{M}$, namely the variations induced by the flow of vectorfields $X$ on $M$ corresponding to sections $\pi(X)=s \in \Gamma Q$.

2. In more detail the concepts are as follows. The foliation $\mathscr{F}$ is given by the exact sequence of vector bundles $0 \rightarrow L \rightarrow T_{M} \stackrel{\pi}{\rightarrow} Q \rightarrow 0$. The splitting $\sigma: Q \rightarrow T_{M}$ defined by $g_{M}$ identifies $Q$ with the orthogonal complement $L^{\perp}$, and induces a metric $g_{Q}$ on $Q$. Similarly, a family $g_{M}(t)$ of metrics on $M$ induces a family of metrics $g_{Q}(t)$ on $Q$ with $g_{Q}(t)\left(s, s^{\prime}\right)=g_{M}(t)\left(\sigma s, \sigma s^{\prime}\right)$ for $s, s^{\prime} \in \Gamma Q$. The energy of $\mathscr{F}$ was defined in [KT] on a compact oriented $M^{n}$ by

$$
E(\mathscr{F})=\frac{1}{2}\|\pi\|^{2}=\frac{1}{2} \int_{M} g_{Q}(\pi \wedge * \pi)
$$

where the integrand is obtained from the pairing of forms

$$
\Omega^{r}(M, Q) \otimes \Omega^{n-r}(M, Q) \rightarrow \Omega^{n}(M, \mathbf{R})
$$

defined by the exterior product and the pairing $g_{Q}: Q \otimes Q \rightarrow \mathbf{R}$. More generally, let

$$
E(\mathscr{F})_{t}=\frac{1}{2}\|\pi\|_{t}^{2}=\frac{1}{2} \int_{M} g_{Q}(t)(\pi \wedge * \pi) .
$$

Received by the editors April 11, 1985 and, in revised form, September 30, 1985.

1980 Mathematics Subject Classification (1985 Revision). Primary 57R30; Secondary 58D17, 58E11, $58 \mathrm{E} 20$.

${ }^{1}$ Work supported by a grant from the National Science Foundation. 
Let now more particularly $X$ be a vectorfield on $M$ and $g_{M}(t)=\pi_{t}^{*} g_{M}$ the pull-back of $g_{M}$ by the flow $\phi_{t}$ of $X$. This defines $E(\mathscr{F})_{t}$ by formula (2). The result to be proved is now stated as follows.

THEOREM. Let $\mathscr{F}$ be a Riemannian foliation on the compact oriented Riemannian manifold $\left(M, g_{M}\right)$. Then $\mathscr{F}$ is harmonic (equivalently all leaves of $\mathscr{F}$ are minimal) iff $d /\left.d t\right|_{t=0} E(\mathscr{F})_{t}=0$ for all variations $g_{M}(t)=\phi_{t}^{*} g_{M}$, where $\phi_{t}$ is the flow of a vectorfield $X$ on $M$.

More precisely, we have the formula

$$
\left.\frac{d}{d t}\right|_{t=0} E(\mathscr{F})_{t}=\langle s, \tau\rangle
$$

for the global scalar product of $s=\pi(X) \in \Gamma Q$ with the tension $\tau \in \Gamma Q$ of $\mathscr{F}$.

We recall the definition of the tension [KT]. Let $W(s): L \rightarrow L$ be the Weingarten map of the foliation $\mathscr{F}$, defined for $s \in \Gamma Q$ by the formula

$$
W(s) Y=-\pi^{\perp}\left(\nabla_{Y}^{M} \sigma(s)\right) \text {, }
$$

where $\pi^{\perp}: T M \rightarrow L$ denotes the orthogonal projection. Then $\tau \in \Gamma Q$ is characterized by $\operatorname{Tr} W(s)=g_{Q}(s, \tau), s \in \Gamma Q$. It is (up to a factor $1 / p$ ) the mean curvature vectorfield of the leaves of $\mathscr{F}$ in $\left(M, g_{M}\right)$, and so the leaves of $\mathscr{F}$ are minimal precisely when $\tau=0$.

3. PROOF OF THE THEOREM. We have to calculate

$$
\left.\frac{d}{d t}\right|_{t=0} E(F)_{t}=\left.\frac{1}{2} \int_{M} \frac{d}{d t}\right|_{t=0} g_{Q}(t)(\pi \wedge * \pi) .
$$

Now let $B_{\pi}(t): T M \rightarrow T M$ be defined by

Then $B_{\pi}(t)$ is linear, and

$$
g_{Q}(t)(\pi(X), \pi(Y))=g_{M}(0)\left(B_{\pi}(t) X, Y\right) .
$$

$$
g_{Q}(t)(\pi \wedge * \pi)=\operatorname{Tr} B_{\pi}(t) \cdot \mu_{M}, \quad \mu_{M}=\text { Riemannian volume form. }
$$

We evaluate the RHS in a neighborhood of $x \in M$ on a local $g_{M}$ orthonormal frame $E_{A}(A=1, \ldots, n)$ of $T M$. For our purpose it is enough to assume that the basis $e_{A}=\left(E_{A}\right)_{x}$ of $T_{x} M$ is such that the $e_{i}(i=1, \ldots, p)$ form a basis of $L_{x}$ and the $e_{\alpha}$ $(\alpha=p+1, \ldots, n)$ a basis of $L_{x}^{\perp} \cong Q_{x}$. Then

$$
\begin{aligned}
\left.\frac{d}{d t}\right|_{t=0} \operatorname{Tr} B_{\pi}(t) & =\left.\frac{d}{d t}\right|_{t=0} \sum_{A=1}^{n} g_{M}\left(B_{\pi}(t) E_{A}, E_{A}\right) \\
& =\left.\frac{d}{d t}\right|_{t=0} \sum_{A} g_{Q}(t)\left(\pi\left(E_{A}\right), \pi\left(E_{A}\right)\right) \\
& =\sum_{A} \lim _{t \rightarrow 0} \frac{1}{t}\left(g_{Q}(t)-g_{Q}(0)\right)\left(\pi\left(E_{A}\right), \pi\left(E_{A}\right)\right) \\
& =\sum_{\alpha} \lim _{t \rightarrow 0} \frac{1}{t}\left(\left(\phi_{t}^{*} g_{M}\right)-g_{M}\right)\left(E_{\alpha}, E_{\alpha}\right) \\
& =\sum_{\alpha}\left(\theta(X) g_{M}\right)\left(E_{\alpha}, E_{\alpha}\right)=\sum_{\alpha}\left\{X g_{M}\left(E_{\alpha}, E_{\alpha}\right)-2 g_{M}\left(\left[X, E_{\alpha}\right], E_{\alpha}\right)\right\} \\
& =\sum_{A}\left\{X g_{Q}\left(\pi\left(E_{A}\right), \pi\left(E_{A}\right)\right)-2 g_{Q}\left(\pi\left[X, E_{A}\right], \pi\left(E_{A}\right)\right)\right\} .
\end{aligned}
$$


Next we use the canonical metric and torsion free connection $\nabla$ in $Q$, given explicitly by the formula [KT, 1.3]

$$
\nabla_{X} s= \begin{cases}\pi[X, \sigma(s)] & \text { for } X \in \Gamma L, s \in \Gamma Q \\ \pi\left(\nabla_{X}^{M} \sigma(s)\right) & \text { for } X \in \Gamma L^{\perp}, s \in \Gamma Q\end{cases}
$$

The holonomy invariance of $g_{M}$ implies

$$
X g_{Q}\left(\pi\left(E_{A}\right), \pi\left(E_{B}\right)\right)=g_{Q}\left(\nabla_{X} \pi\left(E_{A}\right), \pi\left(E_{B}\right)\right)+g_{Q}\left(\pi\left(E_{A}\right), \nabla_{X} \pi\left(E_{B}\right)\right) .
$$

Substituting this in the previous formula yields

$$
\begin{aligned}
\left.\frac{d}{d t}\right|_{t=0} \operatorname{Tr} B_{\pi}(t) & =2 \sum_{A}\left\{g_{Q}\left(\nabla_{X} \pi\left(E_{A}\right), \pi\left(E_{A}\right)\right)-g_{Q}\left(\pi\left[X, E_{A}\right], E_{A}\right)\right\} \\
& =2 \sum_{A} g_{Q}\left(\nabla_{E_{A}} \pi(X), \pi\left(E_{A}\right)\right),
\end{aligned}
$$

where the last equality follows from the vanishing of the torsion $T_{\nabla}$ of $\nabla$ :

$$
T_{\nabla}\left(X, E_{A}\right)=\nabla_{X} \pi\left(E_{A}\right)-\nabla_{E_{A}} \pi(X)-\pi\left[X, E_{A}\right] .
$$

Evaluating at $x \in M$, and using $\pi\left(E_{i}\right)_{x}=\pi\left(e_{i}\right)=0(i=1, \ldots, p), \pi\left(E_{\alpha}\right)_{x}=e_{\alpha}$ $(\alpha=p+1, \ldots, n)$, we obtain

$$
\left(\left.\frac{d}{d t}\right|_{t=0} \operatorname{Tr} B_{\pi}(t)\right)_{x}=2 \sum_{\alpha=p+1}^{n} g_{Q}\left(\nabla_{e_{\alpha}} \pi(X), e_{\alpha}\right) .
$$

But for $s=\pi(X)$ the scalar product of $\nabla s, \pi \in \Omega^{1}(M, Q)$ at $x$, is given by

$$
g_{Q}(\nabla s, \pi)_{x}=\sum_{A} g_{Q}\left(\nabla_{E_{A}} s, \pi\left(E_{A}\right)\right)_{x}=\sum_{\alpha} g_{Q}\left(\nabla_{e_{\alpha}} s, e_{\alpha}\right) \text {. }
$$

Summarizing, we then have

$$
\begin{aligned}
\left.\frac{d}{d t}\right|_{t=0} E(\mathscr{F})_{t} & =\left.\frac{1}{2} \int_{M} \frac{d}{d t}\right|_{t=0} \operatorname{Tr} B_{\pi}(t) \cdot \mu_{M}=\int_{M} g_{Q}(\nabla s, \pi) \cdot \mu_{M} \\
& =\langle\nabla s, \pi\rangle=\left\langle d_{\nabla} s, \pi\right\rangle=\left\langle s, d_{\nabla}^{*} \pi\right\rangle,
\end{aligned}
$$

where $d_{\nabla}: \Omega^{r}(M, Q) \rightarrow \Omega^{r+1}(M, Q)$ is the covariant exterior derivative associated to $\nabla$, and $d_{\nabla}^{*}$ its adjoint (with respect to the global scalar product $\langle$,$\rangle ). Since$ $\tau=d_{\nabla}^{*} \pi$ [KT, Proposition 3.2], the desired formula (3) follows.

We have already noted that the vanishing of $\tau$ means that all leaves are minimal. We observe further that $\Delta \pi=0$ for the Laplacian $\Delta$ associated to $d_{\nabla}, d_{\nabla}^{*}$, which is the reason why these foliations were called harmonic in [KT]. In that paper, many examples of such foliations are discussed. For a foliation defined by a submersion with connected inverse images, the terminology coincides with the harmonic maps terminology of [EL, ES].

4. In order to emphasize the restricted nature of the variations $g_{M}(t)$ in the theorem just proved, it is of interest to comment on the calculation of the first variation in the case of an arbitrary family of metrics $g_{M}(t)$. For this purpose it is useful to describe the path $g_{M}(t)$ in the space $\mathscr{M}$ of Riemannian metrics on $M$ following Ebin's slice theorem [BE, E]. Let $\mathscr{D}$ be the diffeomorphism group of $M$, 
acting on $\mathscr{M}$ by pull-back. Then a neighborhood of $g_{M}(0)=g$ in $\mathscr{M}$ can be diffeomorphically represented by a product $U \times S$ as follows. Let $I_{g} \subset \mathscr{D}$ be the isotropy group of $g$. There exists a neighborhood $U$ of $g$ in $\mathscr{D} / I_{g}$, a slice $S$ through $g$ of the $\mathscr{D}$-action transversal to the $\mathscr{D}$-orbit of $g$, and a section $\eta: U \rightarrow \mathscr{D}$ of the quotient map $\mathscr{D} \rightarrow \mathscr{D} / I_{g}$ such that $\eta^{*}(u) s$ gives a product representation $U \times S$ of a neighborhood of $g$ in $\mathscr{M}$. For an arbitrary family $g_{M}(t)$ with $g_{M}(0)=g_{M}$ we then have $g_{M}(t)=\eta(t)^{*} s(t)$ with $s(0)=g_{M}$ and $\eta(0)=\mathrm{id}$. This implies with notations as before

$$
\begin{aligned}
\left.\frac{d}{d t}\right|_{t=0} E(\mathscr{F})_{t}= & \left.\frac{1}{2} \int_{M} \frac{d}{d t}\right|_{t=0} \sum_{A=p+1}^{n}\left(\eta^{*}(t) s(t)\right)\left(E_{A}, E_{A}\right) \cdot \mu_{M} \\
= & \frac{1}{2} \int_{M} \sum_{A=p+1}^{n} s(0)\left(E_{A}, E_{A}\right) \cdot \mu_{M} \\
& +\left.\frac{1}{2} \int_{M} \frac{d}{d t}\right|_{t=0} \sum_{A=p+1}^{n} s(0)\left(\eta(t)_{*} E_{A}, \eta(t)_{*} E_{A}\right) \cdot \mu_{M} .
\end{aligned}
$$

The second contribution can be expressed in terms of the vectorfield $X=$ $d /\left.d t\right|_{t=0} \eta(t)$ in the form

$$
\frac{1}{2} \int_{M} \sum_{A=1}^{n}\left(\theta(X) g_{M}\right)\left(E_{A}, E_{A}\right) \cdot \mu_{M}
$$

whose vanishing (for all possible $X$ ) is equivalent to the harmonicity of $\mathscr{F}$ by our result. But there is a further contribution towards the first variation depending on the symmetric bilinear form $\dot{s}(0) \in T_{g} S$, the tangent space at $g_{M}=g$ to the slice $S$. This tangent space is well described in [E] as a subspace of the space of symmetric bilinear forms on $M$, and the contribution towards the first variation arising from $\dot{s}(0)$ can be nontrivial, even for a harmonic foliation $\mathscr{F}$.

\section{REFERENCES}

[BE] M. Berger and D. Ebin, Some decompositions of the space of symmetric tensors on a Riemannian manifold, J. Differential Geom. 3 (1969), 379-392.

[E] D. Ebin, The manifold of Riemannian metrics, Proc. Sympos. Pure Math., vol. 15, Amer. Math. Soc., Providence, R. I., 1970, pp. 11-40.

[EL] J. Eells and L. Lemaire, A report on harmonic maps, Bull. London Math. Soc. 10 (1978), 1-68.

[ES] J. Eells and J. H. Sampson, Harmonic mappings of Riemannian manifolds, Amer. J. Math. 86 (1964), 109-160.

[KT] F. W. Kamber and Ph. Tondeur, Harmonic foliations, Proc. NSF Conf. on Harmonic Maps, Tulane 1980, Lecture Notes in Math., vol. 949, Springer-Verlag, Berlin and New York, 1982, pp. 87-121.

[R] B. L. Reinhart, Foliated manifolds with bundle-like metrics, Ann. of Math. (2) 69 (1959), 119-132.

[S] D. Sullivan, A homological characterization of foliations consisting of minimal surfaces, Comment. Math. Helv. 54 (1979), 218-223.

Department of Mathematics, Michigan State University, East Lansing, Michigan 48824

Department of Mathematics, University of Illinois, 1409 West Green Street, Urbana, ILLINOIS 61801 